\title{
Advancements in the Evolution of Human Capacities to Know
}

Mark C Pharoah

Abstract: The premise of this paper is that there are three distinct and hierarchical 'categories of knowledge' (Pharoah 2018). The first of these is physiological knowledge which is acquired over generations through the interaction between replicating lineages and the environment. This interaction facilitates the evolution of meaningful physiological structures, forms, functions, and qualitative ascriptions. Second, there is phenomenal knowledge which is qualified by the utilisation of real-time experience to effect an individuated spatiotemporal subjective perspective. This capability requires sophisticated cognitive capabilities. Conceptual knowledge is the third category and constitutes a network of abstracted principles about the spatiotemporal and phenomenal world of experience. From this starting premise, I argue that human knowledge can still be viewed as impoverished because of the absence of the next category which has not yet emerged. I suggest that this category will be apparent when a fuller understanding is acquired concerning the dynamic nature of concept construction and structuring. This will demand a transdisciplinary and multimodal approach.

Keywords: Conceptual knowledge, phenomenal knowledge, physiological knowledge, ontological hierarchy, discourse, equilibria.

\section{INTRODUCTION}

There was once a man who fell asleep while sitting in an armchair in front of his living room fire. To his horror, he woke to find that his feet had been badly damaged by the fire. They had been too close, but he had felt nothing: some years before, he had become paralysed in a motorcycle accident-he was now 'blind' to the sensation of pain in his legs and feet. I also recall an occasion when I locked eyes with a gorilla in a zoo. There seemed to be a certain understanding between us-a shared experience maybe. But there was also an unfathomable depth of puzzlement, or unknowing. In a similar vein, I recall seeing an insect, with apparent speed of purpose, traversing the great expanse of my living room floor in blissful ignorance that it was in an inhospitable desert with no hope of survival. In each case there is knowing and yet an absence of knowing.

I am mentioning each of these occasions by way of introduction to the possibility that humanity is actually also blind to a certain way of knowing: humans have a certain blindness in much the same way as the insect, the ape or the paralysed man. In each example, it is not known by the subject that there is an unknowing -think of the insect or the ape who appear not to know of the 'wider picture'. And the paralysed man too is tragically unknowing of the situation before him. In this paper, I will make the case that it is within humanity's capabilities to know reality on a different ontological plane; humanity need not have the blindness.

\section{BACKGROUND}

Pharoah 2018 talks of three differentiated (ontologically distinct) classes of interactive discourse. Each class determines an entirely distinct category of meaningful relation between the interacting subject and its world. The overall picture he proposes is that the meaningful relations in each class qualify distinct categories of knowing about the world. Those classes of which he speaks are the physiological, the phenomenal, and the conceptual. 
In relation to the physiological, replication facilitates a generational discourse which determines a meaningful lineage-environment correspondence. This results in the evolution of biochemical capacities that assimilate environmental particulars in such a way that determines a unique and meaningful physiological knowledge category. Importantly, this meaning includes the qualitative ascription to the physical. All innate behaviours are informed by this category of knowing which has evolved over many generations.

In the case of the phenomenal, neural mechanisms have the capability to prioritise the individual's biochemically assimilated environment. This characterises a meaningful and constantly changing experiential discourse. The individual creature thereby possesses a qualitatively delineated spatiotemporal world-view that qualifies the character of its phenomenal experience. This process defines a uniquely subjective phenomenal knowledge category which evolves from one experiential moment to the next.

Thirdly, with the conceptual, cognitive mechanisms facilitate the development of abstracted principles concerning a spatiotemporal and rule-bound world. This generates an introspective inquiry concerning the existential nature of the subject's relationship with the world and qualifies a conceptually constructed knowledge category which informs intrapersonal, cultural and social stances, prejudices and ideologies.

Each category in the hierarchy requires the implementation of a distinctive mechanism of environmental engagement. In effect, there is an emergent hierarchy of evolving categories delineated by the nature of their mechanism of environmental engagement. Each mechanism of environmental engagement justifies its existence, importantly, where it bears some pertinent meaningful correspondence with the environment.

In Pharoah's (2018) summary, he goes on to state:

To say there is a hierarchy implies that it [the hierarchy] probably extends in both directions to more basal and to higher levels - I have detailed only three levels in this paper, namely, the physiological, phenomenal and conceptual. But by extension, one can surmise that in the downward direction we have the precursor to replicating forms whose ontological distinctions are described by classical physics and, a level further down, by quantum physics. In the opposite upward direction, we can surmise that there is likely to be another category in the hierarchy that is yet to emerge from the human existential world-view. (Pharoah 2018, 443)

So if we are talking of distinct classes of knowing, Pharoah is saying that the hierarchical nature of his model indicates that there is another class of knowing that extends beyond the conceptual to which humans are currently ignorant (or blind)-as intimated in the introduction.

In the cases of the insect, ape, and paralysed man, there is some kind of knowing between the subject and the object of experience that is diminished or impoverished. What Pharoah indicates is that humans also have an impoverished world-view because the next ontologically delineated hierarchical level has not yet emerged.

\section{LANGUAGE AND DISCOURSE}

Language is not mentioned in Pharoah's paper. Rather he talks about discourse in the broadest terms: "In my view, meaning cannot be acquired without some kind of mechanism of discourse, where a mechanism of discourse is one that is capable of assigning value..." (431). His argument is that discourse can be said to take place where any kind of interactive mechanism creates meaning whose value is justified in some way.

Under this broad definition, discourse does not predetermine 'that which is communicated'. Rather, 'that which is communicated', namely 'meaning', is qualified by some process of justification. Importantly, this justification process is unconnected to the mechanism of discourse itself (cf. Putnam 2008 on the view that discourse and communication are separate but interrelated constructs). What this means is that when an agent engages in interactive discourse, it cannot control the character of the meaningful outcome. This is true of all the different types of communication in the proposed hierarchy.

In its broader interpretation, all categories of discourse require a mechanism that makes interaction possible, and a vehicle of transmission. For instance, DNA, through the mechanism of replication, is the principle vehicle that makes possible the discourse between a replicating lineage and the environment over a generational timeline. Note, there is no implicit meaning 'carried' by DNA. The meaning is qualified by a separate uncontrolled process of validation and justification. This maintains a kind of 'ontological divide,' or perhaps a causal divide, between the genotype and the phenotype.

In the case of phenomenal experience, neural mechanisms (mostly unidentified to science) facilitate a real-time experiential discourse. The vehicle of transmission is the individual's entire afferent-efferent neural system which engages the environment. The meaningful subjective world-view of the individual creature is a reflection of the impact of the environment-creature discourse which includes the perceptual-behavioural complex. Once again, a creature's phenomenal knowledge, in its entirety, is qualified by a separate uncontrolled process of validation and justification that maintains the divide between phenomenal content and the functions of the neural network.

In each case, what might be understood as 'that which is communicated through interactive discourse' takes on a very different category of meaning. Interestingly, the communication is both internal, that is, maintained as part of the construct of the subject (or agent), and external to the subject, where 'external' means that it extends into the wider environment. So there is this bifold idea of a construct that perpetuates over time, and 
a fluid open discourse. Additionally, there is the crucial notion of a divide between the mechanism of discourse and that which validates.

Human language is a more familiar example. Languages are thought of as a form of communication: communicating is the purpose of language. But human languages are primarily an evocation of an individual's introspective interpretations of reality (or a rendition of the individual's 'cognitive schemata'), where the communication itself is only part of those introspective considerations and interpretations. Language becomes part of the vehicle of introspective discourse when there are other humans with whom those thought processes can be shared and discussed-noting the broader significance of introspective creativity and social semiotics (Hodge \& Kress 1988). Discourse is the introspective activity, while language is the vehicle which promotes meaningful engagement, both internally and externally. But, as with the other examples, the activity and the vehicle do not determine the meaning-information is not something that is transferred from one to the other. There is an indeterminacy of meaning (notably, this applies not just to translation but to discursive language generally-consider Zolyan 2017). In other words, the meaning is, ultimately, an approximated contextual construct that is validated and justified by separate and unconnected processes. One example of a formalised process of validation is the scientific method. Of course, the extensive body of existing scientific knowledge and its evolutionary development is not controlled by our personal introspections and utterances. This fact conforms to the idea that there is an ontological/causal divide that undoubtedly frustrates all those wishing to steer the course of human knowledge and development.

\section{EQUILIBRIA AND STABILITY}

Pharoah does not mention in his paper the role of equilibria and stability either. But it is an important consideration if one is to explore the next hierarchical level of knowing to which humanity is currently blind:

One of the principle ideas of any state of equilibrium is that it remains in equilibrium until some conflicting impulse motivates change. If we apply this idea to discourse and justification, we can note that when meaning is justified by some process, then that meaning represents a stable truism that remains so until such a time that it is no longer the case. In this way, we can consider different classes of knowledge as being meaningfully coherent and an accurate reflection of reality, and in being so, to be stable (or, to be in a state of equilibrium). This remains the case until such time as the reliability of that meaningful knowing becomes challenged or irrelevant. A subsequent rebalancing of stability is necessarily indicative of the degree to which meaning requires recalibration. In this way, meaning acquisition is not motivated by incompleteness per se (Deacon 2012), for which there is no absolute authority, but by instability.
For each category-those being the physiological, the phenomenal and the conceptual-this variation in the degree (or extent) of instability is reflected in the rate of physiological, behavioural, and introspective adaptation, respectively. With each, there is a commensurable evolutionary gradient that reflects the dynamism of the changing environment-agent relation that is particular to each of the respective classes ('agent' here refers to the replicating lineage, the individual creature, or the human's conceptual stance, respectively).

Notably, to some extent all equilibria resist destabilising influences. Inevitably, however, for each class, stability is always being challenged and evolutionary adaptation takes place, be it physiological, behavioural, or conceptual. Consequently, a species will tend to evolve when conditions make prescient survival demands (Kashtan et al. 2007); a creature will adapt its behaviours (i.e. to learn) more readily when conditions undermine what it understands to be the case; a human will tend to think more broadly when the coherence of its stance is destabilised and a stable transitional alternative comes into focus.

And, as was mentioned previously, the justification that controls the evolution of meaning, in each class, is not controlled by the agent: a species does not control its evolution; a creature does not control what it learns; a human being does not control its conceptual stance or human knowledge. But in each case, what the agent does, does affect the dynamic of evolutionary adaptation (broadly construed) according to a complex dynamic of influences and effects (consider Sharov 2016, 2017 on the reciprocal influence on evolution of agent scaffolding and constraints).

\section{MOVING FORWARD TO THE NEXT HIERARCHICAL CATEGORY OF KNOWING REALITY}

In each class, a distinct category of knowing evolves in a way that is uncontrolled by the agent because the process that justifies the validity of that meaning is separate. What is notable, however, is that the emergence of each new level leads to the capability to control what was previously uncontrolled at the level below. Let us look at each level in turn:

While the individuals of species possess innately acquired physiological knowledge, they remain incapable of controlling the behaviours which are informed by their innate inclinations. But, at the next hierarchical level (the phenomenal), the individual has gained the capacity to moderate its innate inclinations in virtue of being able to learn from experience; it can control what previously was not controlled (namely, its behavioural responses to varying environmental conditions).

Although such creatures do learn from experience, they cannot control the learning process itself; they do not control what they learn. But with the emergence of the next hierarchical level (the conceptual), the individual 
human being has gained the capacity to control the learning process. It does this by being creative in its application to learning. This is what abstract introspection facilitates; it facilitates a control over the learning process. Human behaviour reflects this capability.

Even so, humans are not able to control or harness creativity in a way that controls the dynamic of its introspections and the dynamic of human knowledge acquisition (Rozov 2012). The human is blind to this capability. I propose that the emergence of the next level in the hierarchy will make these controls possible.

Importantly, this capability to harness or control the dynamic of human introspection and knowledge acquisition is not just a different way of organising knowledge, of systemising human endeavour or of structuring information. It is not a simple change in the way we operate either as humans or as a global society. Rather, it is an ontologically distinct capability: a revelatory way of being in the world and observing reality. It is a new kind of meaning and knowledge about reality. "Humanity's steering of the future must involve going beyond humanity in some sense" (Rickles 2016, 49).

\section{CONCEPTUAL KNOWLEDGE}

So, we as humans are on one side of a divide, and on the other side is the goal. In a way, we are like the ape in the zoo looking into the eyes of the future of humanity: "There seems to be a certain understanding between us, a shared experience maybe. But there is also an unfathomable depth of puzzlement, or unknowing." Where do we go from here?

What is required is a significant transdisciplinary program of theoretical research (Polis 1993). When we say of humans that they strive for knowledge, what we really mean is that they strive for an understanding of the nature of reality that is conceptual in construction. Each of us has an ideological stance to the world that is constructed from a network of conceptual principles that, in turn, are incorporated into social forms (Pólos et al. 2002). In my view then, we need to start with 'the concept'. We need to examine the dynamic of concept formation and construction and determine how they relate to each other in a network of connective principles about reality. For instance, some concepts are restricted by the boundaries of our phenomenal knowledge and our innate physiological knowledge. Others reflect the nature of our social discourse and communications. Some conceptual apparatuses are centred around maintaining a stable view of the world and our place in it and, from there, connect with our emotions. Many, such as those formulated in mathematics and logic, are pure abstractions-abstractions founded on quantitative and qualitative axioms. Seen together, they form a whole. They form a whole that qualifies the construct of the individual self-as it 'sees' itself and the world in which it exists. It is this 'seeing' that can be augmented and extended.

Understanding the different categories of concept and their dynamic relationships will give insights into what determines ideological equilibria. Such an understanding will determine the root knowledge underpinning the ideology of prejudice, bias, tolerance, and creativity, thereby heightening any given individual human's ability to 'see' the mind of her/his fellow human and interpret their contribution to a global society. The ultimate goal would be to enable humanity to steer the evolution of human knowledge in a unique and positive way.

\section{REFERENCES}

Deacon, T., 2012. Incomplete nature: How mind emerged from matter. New York, NY: Norton.

Hodge, R., G. Kress., 1988. Social semiotics. Cambridge: Polity.

Kashtan, N., Noor, E., Alon, U., 2007. Varying environments can speed up evolution. Proceedings of the National Academy of Sciences, 104(34), 13711-13716.

Polis, D., 1993. Paradigms for an open philosophy. Metaphilosophy, 24(1/2), 33-46.

Pólos, L., Hannan, M. T., Carroll, G. R., 2002. Foundations of a theory of social forms. Industrial and Corporate Change, 11(1), 85-115.

Putnam, L., 2008. Images of the communication-discourse relationship. Discourse and Communication, 2(3), 339-345.

Rickles, D., 2016. A participatory future of humanity. In Aguirre, A., Foster, B., Merali, Z. (Eds.), How should humanity steer the future? The Frontiers Collection. Cham, Switzerland: Springer, pp. 49-59.

Rozov, N. S., 2012. The role of imagination in socio-historical development: The ontology of modernization mechanisms. METOD: Moscow Social Sciences Yearbook, 3.

Sharov, A., 2016. Evolution of natural agents: Preservation, advance, and emergence of functional information, Biosemiotics 9(1), 103-120.

Sharov, A., 2017. Composite agency: Semiotics of modularity and guiding interactions. Biosemiotics, 10(2), 157-178.

Shmerlina, I. A., 2019. 'Social form' as a subject and a tool of sociological analysis: historical and scientific introduction. METOD: Moscow Social Sciences Yearbook, 9.

Zolyan, S. T., 2017. The indeterminacy of translation as a projection of the dynamic semantics of text. METOD: Moscow Social Sciences Yearbook, 7.

Return to front page $\uparrow$ 